\title{
Recognition and Applications of Emotion Detection in Driving Fatigue
}

\author{
Bi Hongzhe and Shen Gongzhang*
}

School of Automation Science and Electrical Engineering, Beihang University Beijing 100191, China

\begin{abstract}
For the driver at the long-term driving or at the poor mental state, the emotion based driving fatigue recognition algorithm is proposed, and we use the algorithm to effectively prevent the continuous driving of the driver in the state of fatigue, so as to avoid the accident. We sample the facial images of the driver and establish their feature space, and compare them with the fatigue training samples, achieve in real time the driving category attributes of the driver. According to the driving category attributes, we judge whether the driver is in the state of fatigue so as to appropriately intervene in the
\end{abstract} behavior of the driver.

Keywords: Driving fatigue, eye tracking, blink frequency, principal component extraction.

\section{INTRODUCTION}

According to statistics on main reason of road traffic accidents from the related departments, driving fatigue accounts for the largest proportion. Many drivers in the state of fatigue often may be sleepy all the time, even experience a short loss of consciousness, and they often do not know they are already in the drowsy state. Overfatigue is referred to that the driver drives more than eight hours in a day or exerts on excessive physical in other work or is lack of sleep, so that the driver is sleepy and weakness in driving, and cannot found and accurately handle road traffic problems in the PRC Road Management Regulations granted by Ministry of Public Security. According to this concept, fatigue driving is confirmed by one of two points: Firstly, cumulative time of driver to drive the vehicle is calculated within 24 hours before the fatigue behavior found, if it is more than eight hours, even though the diver has no external appearance of fatigue driving; Secondly, through the investigation, driver is confirmed to be excess physical exertion due to some reasons or lack of sleep, and drive in sleepy and weakness way so that he cannot timely find and accurately process traffic problems, even though the cumulative time is not more than eight hours. Although there are many technical testing methods, only relying on appropriate testing technology using by law enforcement officers is not enough due to the limited human and material resources, and diver lacking of some relevant concepts on fatigue driving. Each year around the world road traffic accidents causes road safety problem, and has been greatly threatened the life and property of social public [1-3]. Among the many traffic accidents, driver fatigue is the main causes; Klauer et al found that the probability of traffic accidents due to driving fatigue is 4 to 6 times compared to normal driving [4], so it has become the focus of global attention. Therefore, research on the related technology of the driving fatigue warning has important practical significance and application value.

The driving fatigue detection methods have the following: driver's physiological signal based methods [5-7], behavior based methods, operation state of vehicle based method [8-10], and facial expressions based methods [11, 12]. Physiological signal based methods requires the sensor in contact with the driver's body and detail requirements on the ways and points of contact, it is unsuitable for universal application of normal driving. Methods based on the driver's head movement have a lot of randomness and chance due to the causal relationship between the specific head movements and fatigue, such as a lane change and swing driving reflecting vehicle driving behavior, although these behaviors partially reflect whether a driver is in a state of fatigue, it is difficult to determine the driver indeed in state of fatigue. Computer vision based methods use camera to capture driver's image in real-time, analyze the images to determine the driver's fatigue state. The method has the characteristics of non-contact, but also need further improvement in terms of the accuracy and practical usefulness. In the paper, image based method proposed obtain the driver's face images in real-time using the infrared light source and an infrared camera, and analyze the eye blink state to determine whether the driver is in the fatigue state. Finally, the integration algorithm is embedded in the devices of the car, we complete preliminary research from the algorithm to practicability and industrialization to the system.

In this paper, we research the detection methods based on the driver's fatigue facial expression, which usually use a camera to obtain facial information and identify facial feature points, and extract fatigue expression feature, then determine whether the driver's in the state of fatigue or not. The advantage of this non-contact detection method does not interfere with normal driving of the driver. The current methods have mainly focused on local feature extraction, such as PERCLOS based on eye movement characteristics, although the recognition rate of the detection method based 


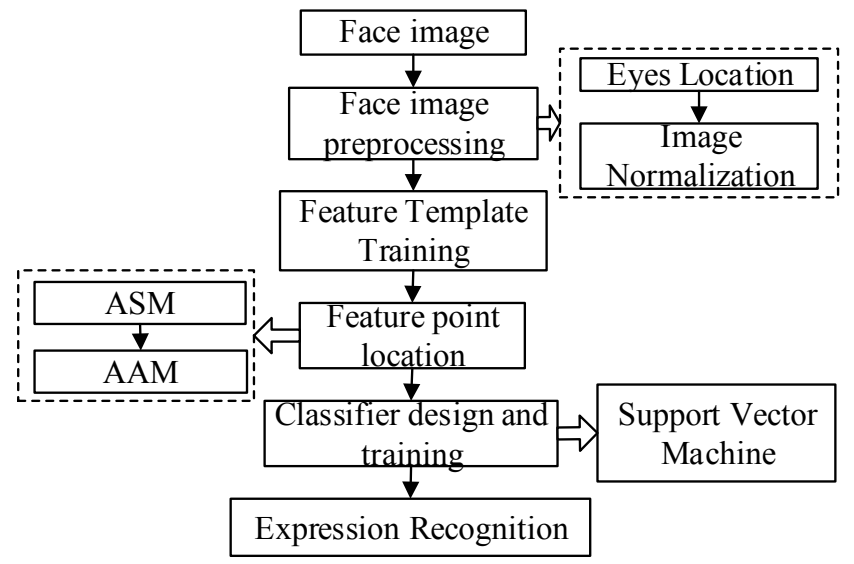

Fig. (1). Overall framework of the system diagram.

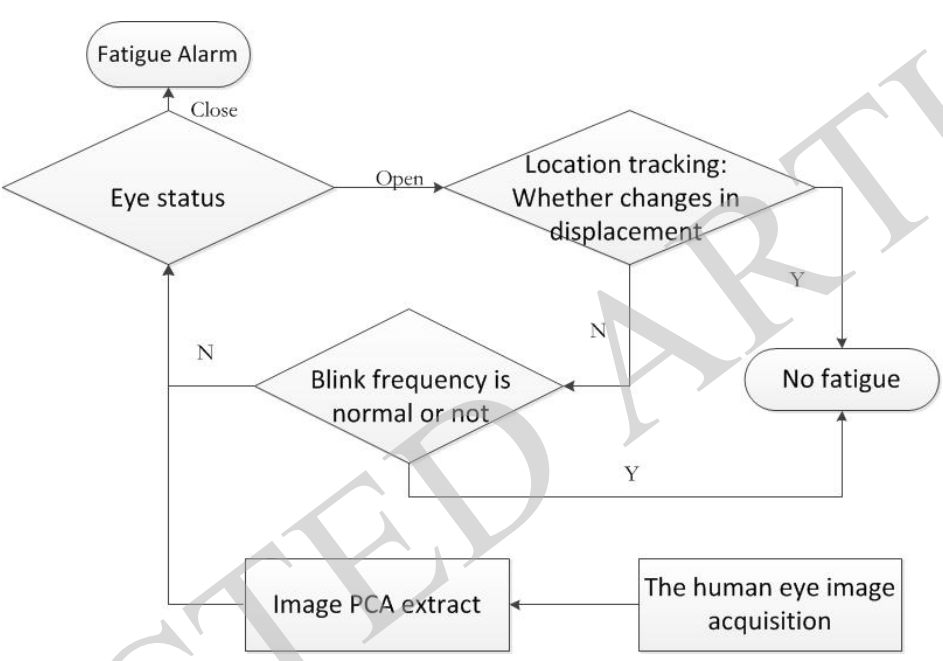

Fig. (2). Diagram of implementation process of driver fatigue warning.

on local feature extraction has reached more than $80 \%$, the overall facial expression feature has not been fully excavation.

So far, many driving fatigue alarm devices are successful developed such as vehicle safety auxiliary driving technologies, but there are few equipment on the use of machine vision for driver fatigue monitoring devices. Machine vision detection based method has the virtues of realtime, accuracy, practicality and economy etc, we fully use the advantages of machine vision, and study to monitor driver in the state of fatigue driving in real-time using machine vision detection based method. This method not only expands the scope of driving fatigue monitoring technologies, but also its further development has important practice.

\section{IMPLEMENTATION PROCESS OF DRIVING FA- TIGUE WARNING}

The judgment process of drivers' eyes state is described as follows: firstly, we capture driver's video. Because the image information is clear or not, it will directly affect the accuracy of the algorithm, the image preprocessing need to be made to provide the standard image format of easily distinguishing the characteristics of face and eye detection. Face detection is a key step because it involves the real-time debugging for the later system; the algorithms selected should be taken the accuracy, realtime and portability aspects into account. Then we use the eye location algorithm to select and track the eye area. In the case of a normal eye movement, judgment of simple blink can improve real-time. Finally, we extract the characteristic parameters of the eyes, judge whether a driver fatigue and send a warning message according to PERCLOS criteria. And some of the existing feature extraction algorithms are used in the eye detection and extraction process, the process is shown in Fig. (1).

The process of fatigue driving warning using judgment of driver's eye state is described as follows: we analyze the video of the driving situation. The driver is under the specific environment, the duration of eyes fixing the target under normal driving is $100 \mathrm{~ms}$ to $350 \mathrm{~ms}$. If a person is in the state of fatigue, we find each point of the eyes has no change in displacement, so we can judge if drive is in the state of fatigue using the displacement of eye ball. Once we detect that the eye is in a zero displacement in a period of time, we can begin the judgment of the frequency of blink, at last it all will return to the judgment of that the eyes are open or closed, the process is showed in Fig. (2). 


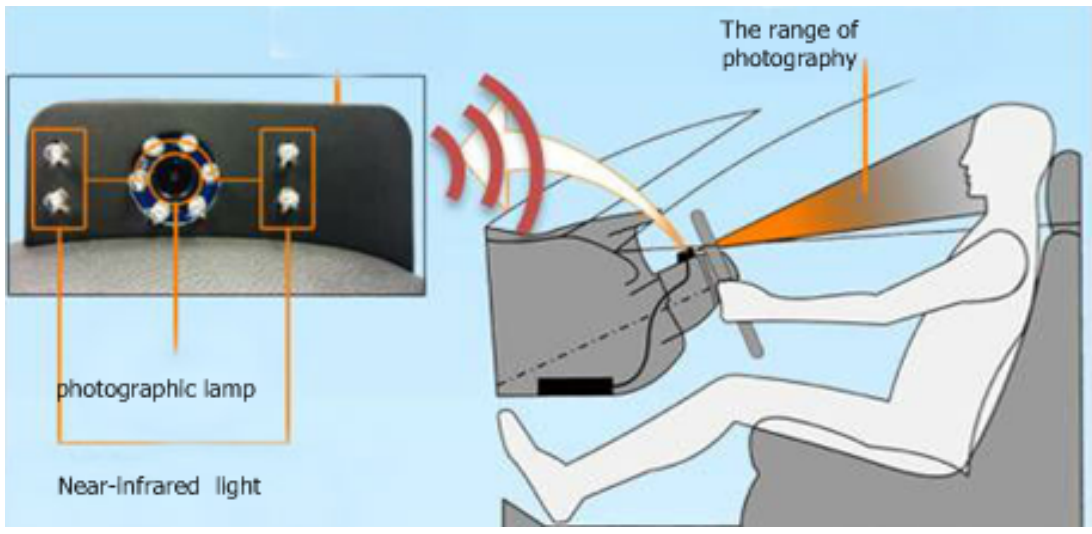

Fig. (3). Implementation of the system.

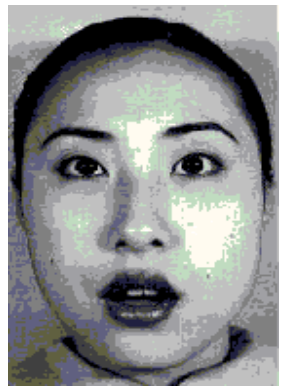

Original Image

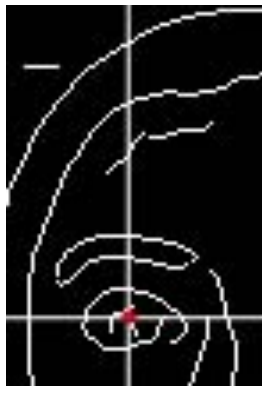

L-eye Projection

Fig. (4). Eye location process.

Implementation of the system: we uses a LED infrared light which wavelength is in the range of $800 \mathrm{~nm} \sim 900 \mathrm{~nm}$ in the system video, camera with $900 \mathrm{~nm}$ single optical filter, system acquisition rate with 80 frame/second, and use sound alarm to warn driving fatigue. The host is installed in the car and camera installed in the root of the steering wheel, which can capture the video of the driver all the day, Implementation process of the system is shown in Fig. (3).

\section{IMPLEMENTATION TECHNIQUES OF DRIVING FATIGUE WARNING}

\subsection{Image Preprocessing}

The driver state need be detected in the whole day and night, we use infrared camera to collect the face images of the driver in the system. Using infrared camera, the driver facial images at night are not only sampled, but also it filters interference of additional light source at different weather and time at day time, and improve the stability of the image. There are obvious differences between the images collected through single-band filter and the images collected under natural light, especially skin color is too bright, and iris become white. So the results of detecting the video images under infrared light using the samples and classifier obtained by samples of the human face and the human eye in natural light are not satisfactory. Under practical conditions, we use the same single-band filter and the infrared light to sample human face images and train the similar sample library, and this can ensure the results the robustness. In this paper, we uses the part of human face sample in the near-infrared face database (POLYU-NIRFD) [13] by The Hong Kong Polytechnic University, the other samples used are self-collected. The samples are self-collected using infrared camera and infrared light source equipped with self-designed driving fatigue device in the system; we simulate driving or real car collect human face samples under various weather conditions and different time of day in real car. Some samples of the sample library are shown in Fig. (4).

\subsection{Face Detection and Localization}

Face positioning is first to do for giving the eye location in order to calibrate correct position of the eye area. Positioning the eye areas in the face image reduces computation of searching the eyes, compared to positioning the eye areas in the whole image, and improves the efficiency of method.

Numerous experiments show that the facial skin characteristics is an effective means of segment human face skin color, at the same time, color information statistics and calculation is simple, fast, it can meet the real-time requirements to a large extent. In order to implement real-time driving fatigue detection, we will use the fast projection detection algorithm based on face skin in the system, namely grey value collection of the accumulative amount of calculation along a certain direction cross section $[14,15]$. 


\subsection{Positioning of Human Eye}

We use two steps to position eyes [16]: coarse positioning and precise positioning of eyes. Coarse positioning of eyes means that it must first find the approximate location of the eyes. Because the grayscale of eyes is different from the skin's color, we determine the approximate location of eyes using the grayscale projection method in grayscale graph. The location of eyes is initially identified, and then precise positioning of eyes is implemented using four neighborhood search algorithm and eye constraints.

(1) Course positioning of the eye

Edge detection is an important method of positioning the human eye. Edge detection operator checks the neighborhood of each pixel and quantifies the gray-scale variation rate and determines the direction.

Edge detection operator checks the neighborhood of each pixel and quantifies the gray-scale variation rate and determines the direction. We uses sobel operator to implement edge detection, consider the each point in the image, obtain weighted grayscale difference of four points which are adjacent to each point on the up, lower, left and right directions, make the weighted difference of each direction as the edge images extracted. Then we make projection of the gray value for the edge image in the horizontal and the vertical direction, and the intersection of row and column with the maximum gray value is the approximate location of the eyes. Then we take the upper half of the edge image of the face, then the interception edge image from the horizontal intermediate position will be divided into two parts, at last make the respectively horizontal and vertical gray projection of edges of left and right eye images to reduce the projection influence made by rotation, and other organs [17]. The coarse positioning process of eyes using sobel operator is shown in Fig. (3).

(2) Precise positioning of the eye

After the image is binarized, it is operated by corrosion expansion; the white points of the central eye are filled [18]. In this binary image, we use search algorithms to find out the connected domain in the initial positioning region of the eye. In the paper, we use four neighborhood algorithms. The adjacency is the basic relationship between pixels in the binary image. Four neighborhoods of a pixel are natural neighbors in horizontal and vertical directions. The four neighborhood consists of four adjacent points, namely, the four pixel neighbors of a pixel with coordinates $(a, b)$ are $(a-1, b),(a, b-$ $1),(a+1, b),(a, b+1)$. Then we judge if the connected domains searched out are eyes according to eyes' features. We analyze the physical structure characteristics of the human face and find that the eye has the following characteristics: 1 $\leq$ eye width / eye height $\leq 4.5 ; 1 / 20$ face wide $\leq$ eye width $\leq 1 / 3$ face width; Eye Center height / face height $\geq 1 / 59$.

Besides, the eyes are close to eyebrows, and gray values of eyes and eyebrows are much small, the connected region searched may be the eyes or eyebrows. But eyebrows are under eyes, therefore, we add a restriction condition to distinguish between the eyes and eyebrows: If the candidate regions are more than 2, we compare the center ordinate of candidate eye areas and take the smaller ordinate as the two eyes.

\section{KEY TECHNOLOGIES IN THE WARNING SYS- TEM}

\subsection{Eye Tracking}

We track difficulty eye due to its rapid movement, in the paper the Unscented Kalman filter (UKF) is used to track eyes to obtain more accurate estimation results [19]. The basic idea UKF is as follows:

$$
\begin{aligned}
& X_{k+1}=F\left(X_{k}, U_{k}\right)+V_{k} \\
& y_{k}=H\left(x_{k}\right)+n_{k}
\end{aligned}
$$

where $x_{k}$ represents the unobserved state, $u_{k}$ is the known input source, $y_{k}$ is measurement observed. Process noise $v_{k}$ affects the entire dynamic system, $n_{k}$ is the measurement noise. Because the eye movement is a high nonlinearity, it is difficult to simulate the dynamics of driver's eye movement. In the tracking system, we simulate the new real eye tracking algorithm based on UKF, and use the following nonlinear equations to model the dynamic movement of the eyes:

$$
\begin{aligned}
& x=x_{0}+v t+0.5 a t^{2} \\
& x_{k+1}^{*}=v_{0}+A \sin \left(\omega_{k} t\right) \\
& a_{k+1}=x_{k+1}^{* *}=A_{k} \omega_{k} \cos \left(\omega_{k} t\right)
\end{aligned}
$$

where the initial values of $x_{0}$ and $v_{0}$ are 0 , acceleration a is selected as a sinusoidal distribution, $A_{k}=0.08 \mathrm{~m} / \mathrm{s}$, and the eye movement state uses Newton's laws of motion, the state transition matrix are as follows:

$$
\begin{aligned}
& F=\left[\begin{array}{cccc}
1 & \Delta t & 0 & 0 \\
0 & 1 & 0 & 0 \\
0 & 0 & 1 & \Delta t \\
0 & 0 & 0 & 1
\end{array}\right] \\
& H=\left[\begin{array}{llll}
1 & 0 & 0 & 0 \\
0 & 1 & 0 & 0
\end{array}\right]
\end{aligned}
$$

where $\Delta t$ is the time from the interval of $k$ to $k+1$.

\subsection{Principal Component Extraction of Block PCA Im- age}

Three important characterization of the eye is completely open, neutral and closure. Each state of eye gets 20 pictures. Keeping 99\% energy of the total sample set of 60 images, we extract their base vector to form subspace. After getting the subspace of local images database, we test the 60 images to 


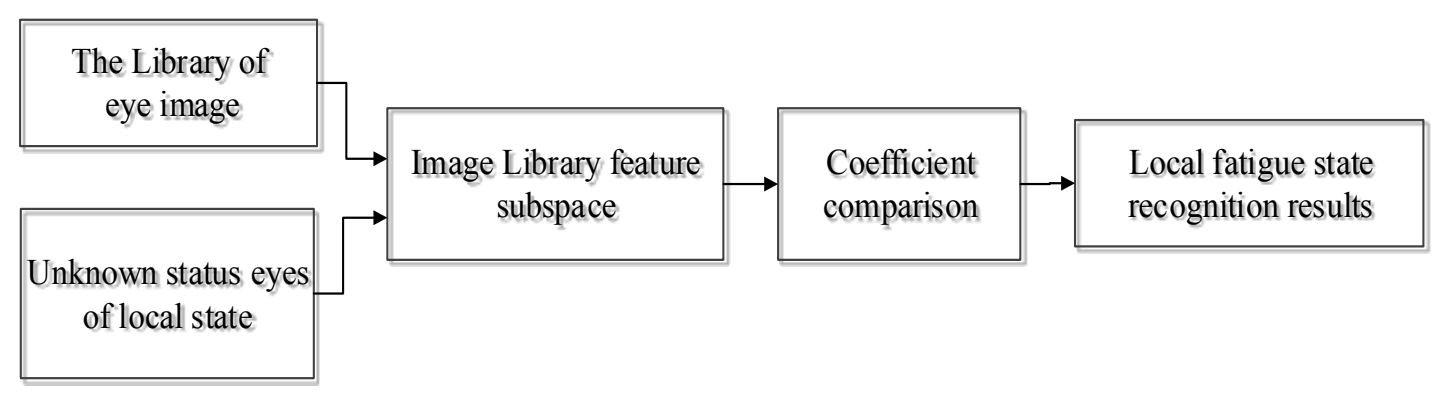

Fig. (5). Identification module of image pca extract.

the 120 samples (40 samples per state) [20]. We measure Euclidean distance and use the nearest neighbor classifier to classify. We use the classification to identify the eyes' state of the face. According to this information, we are easy to detect whether the driver is in the state of fatigue or not. Main recognition process is shown in Fig. (5).

The experimental results show that computation of this method using the block PCA is greatly reduced, and the recognition accuracy is very high.

\subsection{Relationship between Blink Frequency and Degree of Fatigue}

Experiments show that the frames of the continuous eyes closure are many when the driver is in case of exhaustion blink. According to the rough statistics, the frames of eyes' closure is normally $8 \sim 15$, and the frames of the fatigue blink are as much as 100 , and number of actual experiment is much more. From the experiments we can get the approximate relationship of fatigue and blink frequency:

$$
f=(a / x)+b
$$

'a' represents normal blink of a person, commonly 27 beats / $\mathrm{min}, \mathrm{x}$ is the degree of fatigue. Theoretically, the relationship of $\mathrm{f}$ and $x$ is very complex, but in practice we can simplify their relationship through experiments, reasonable with different values for $b$. We can infer that the greater the degree of fatigue detected by the system, the smaller the frequency of blinking. When the degree of fatigue $\mathrm{x}$ continues to increase, the blinking frequency is dropping to almost 0 . For implementation, we can design the timers and counters to record two consecutive blinking time, we got a bunch of data. If a series of consecutive numbers show an incrementing sequence, we can determine the driver is in the state of fatigue.

\section{SIMULATION EXPERIMENTS AND RESULTS}

The combination of using the judgment of eye open or closed state determination, eye's location tracking and the judgment of normal eye blink frequency significantly improves the realtime of system, compared with the only judgment of eyes' state. In the experiments for recognition accuracy, the same action of the person is detected using the fixed camera, the tester's head is always in the middle of image in the video, the head can swing around, look down and upward, etc. The eyes can open, closed and half-closed. The former is defined as system A, the latter is systems B, the test results are shown in Table $\mathbf{1}$ and $\mathbf{2}$.

Table 1. Comparison of frames detected.

\begin{tabular}{|c|c|c|c|}
\hline System & $\begin{array}{c}\text { Total Number of } \\
\text { Frames }\end{array}$ & $\begin{array}{c}\text { Number of Detect- } \\
\text { ed Frames }\end{array}$ & Detection Rate \\
\hline \hline A & 873 & 649 & $74.3 \%$ \\
\hline B & 747 & 536 & $71.8 \%$ \\
\hline
\end{tabular}

Table 2. Recognition rate comparison.

\begin{tabular}{|c|c|c|c|}
\hline \multirow{2}{*}{ Status } & Open & Neutral & Fatigue \\
\hline \hline $\mathrm{A}$ & $83.7 \%$ & $89.7 \%$ & $94.3 \%$ \\
\hline $\mathrm{B}$ & $83.3 \%$ & $77.1 \%$ & $91.7 \%$ \\
\hline
\end{tabular}

\section{CONCLUSION}

The eyes detection method proposed in this paper uses dynamic eye template in the video images to match the image to be tested in order to identify the state of the eye. The method not only effectively avoids confusion of the eyes and eyebrows and the individual differences, but also obtains a good solution to the light interference and other environmental conditions. Meantime, it uses determination of the open and closed state of eyes, positioning, tracking and the judgment of eye blink frequency for normal or not, and the detection domain is much smaller than the whole facial image, and doesn't increase the computation amount. The method has not only ensured the real-time of the system, but also increased in the accuracy rate of the system.

\section{CONFLICT OF INTEREST}

The authors confirm that this article content has no conflict of interest. 


\section{ACKNOWLEDGEMENTS}

Declared none.

\section{REFERENCES}

[1] World Health Organization. Global Status Report on Road Safety: Time for Action. Geneva: http://www.who.int/violence_injury prevention/road_safety_status/2009/en/, 2009.

[2] NHTSA. Traffic Safety Facts. Report DOT HS 811 402, NHTSA, US Department of Transportation, 2011.

[3] L.S. Jin, Q. N. Niu, H. J. Hou, and S.Hu, "A pedestrian detection method using 3D laser scanner," Information An International Interdisciplinary Journal, vol. 15, no. 12A, pp. 5481-5490, 2012.

[4] S. G. Klauer, T. A. Dingus, V. L. Neale, J Sudweeks, and D J Ramsey, The Impact of Driver Inattention on Near Crash/crash Risk: An Analysis Using the 100 Car Naturalistic Driving Study Data, National Highway Traffic Safety Administration, Washington 2006.

[5] I. Takahashi, and K. Yokoyama, Development of a Feedback Stimulation for Drowsy Driver Using Heartbeat Rhythms, Engineering in Medicine and Biology Society, Boston, 2011.

[6] E. C. Murphy, and M. M. Trivedi, "Head pose estimation in computer vision: a survey," IEEE Transactions on Pattern Analysis and Machine Intelligence, vol. 31, no. 4, pp. 607-626, 2009.

[7] X. Fan, Y. Sun, B. Yin, and X. Guo, "Gabor based dynamic representation for human fatigue monitoring in facial image sequences," Pattern Recognition Letters, vol. 31, no. 3, pp. 234-243, 2010.

[8] Y. Kim, K. Youmin, and M. Hahn, "Detecting driver fatigue based on the driver's response pattern and the front view environment of an automobile," In: $2^{\text {nd }}$ International Symposium, pp. 237-240; Osaka, USA, 2008.

[9] P. M. Forsman, B. J. Vila, R. A. Short, C. G. Mott, and H. P. A. V. Dongen, "Efficient driver drowsiness detection at moderate levels of drowsiness," Accident Analysis and Prevention, vol. 50, pp. 341$350,2013$.

[10] F. Friedrichs, and B. Yang, "Drowsiness monitoring by steering and lane data based feathers under real driving conditions," In: $18^{\text {th }}$ European Signal Processing Conference, Aalborg, pp. 23-27, 2010.
[11] I. Garcia, S. Bronte and L. M. Bergasa, "Vision-based drowsiness detector for a realistic driving simulator", In: $13^{\text {th }}$ International IEEE Conference on Intelligent Transportation Systems, pp. 887894, Funchal, 2010.

[12] A. Eskandarian, A. Mortazavi, and R. Akbar Sayed, Drowsy and Fatigued Driving Problem Significance and Detection Based on Driver Control Functions, Handbook of Intelligent Vehicles, Springer, pp. 941-974, 2012.

[13] B.C Zhang, L. Zhang and D. Zhang, "PolyU near-Infrared face database," 2010 http://www4.comp.polyuedu.hk/ biometrics /polyudb_face.htm.

[14] K. Hawari, B. Ghazali, J. Ma, R. Xiao, and S. A. lubis, "An Innovative face detection based on $\mathrm{YCgCr}$ color space," Physics Procedia, vol. 25, pp. 2116-2124, 2012.

[15] Y. H. Chen, K. Hu and S. Ruan, "Statistical skin color detection method without color transformation for real-time surveillance systems," Engineering Applications of Artificial Intelligence, vol. 25, no. 7, pp. 1331-1337, 2012.

[16] Q. Yu and Y. T. Fang., "Multiple perspectives on the human eye sight positioning algorithm," Computer Knowledge and Technology, vol. 7, no. 4, pp. 870-872, 2011

[17] X. M Wang, J. N. Chi. and Z. L. Wang, "Knowledge based on regional characteristics and matching driver eye location method," In: 2009 Chinese Control and Decision Conference Proceedings, China, pp. 3980-3984, 2009.

[18] S. B. Yang, L. Jing and Z. B. Zhang, "Fatigue driving detection method for rapid eye location," Journal of Wuhan Institute of Technology, vol. 35, no. 6, pp. 67-72, 2013.

[19] W. H. Chen and Y. H. Zhao., "The algorithm of improved UKF and the tracking performance goals," Modern Electronic Technology, vol. 34, no. 23, pp. 4-6, 2011.

[20] Y. F. Xia, J. Xiao and H. L. Wu, "The block PCA face recognition of identification confident decision fusion," Computer Engineering, vol. 38 . no. 10 , pp. $148-150,2012$

Received: September 16, 2014

Revised: December 23, 2014

Accepted: December 31, 2014

(C) Hongzhe and Gongzhang; Licensee Bentham Open

This is an open access article licensed under the terms of the Creative Commons Attribution Non-Commercial License (http://creativecommons.org/licenses/by$\mathrm{nc} / 3.0 /$ ) which permits unrestricted, non-commercial use, distribution and reproduction in any medium, provided the work is properly cited. 